\title{
AN INVERSE DYNAMICS APPROACH TO TRAJECTORY OPTIMIZATION FOR AN AEROSPACE PLANE
}

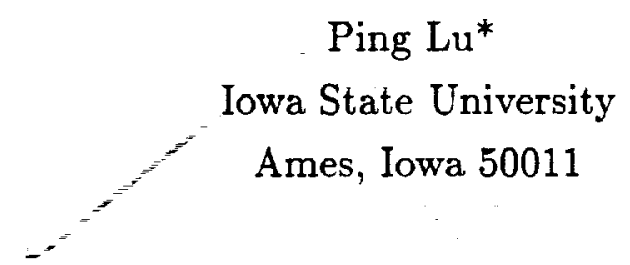

$$
\begin{aligned}
& \text { NNGI-1255 } \\
& 1 N .05 \mathrm{CR} \\
& 131808
\end{aligned}
$$

\begin{abstract}
An inverse dynamics approach for trajectory optimization is proposed. This technique can be useful in many difficult trajectory optimization and control problems. The application of the approach is exemplified by ascent trajectory optimization for an aerospace plane. Both minimum-fuel and minimax types of performance indices are considered. When rocket augmentation is available for ascent, it is shown that accurate orbital insertion can be achieved through the inverse control of the rocket in the presence of disturbances.
\end{abstract}

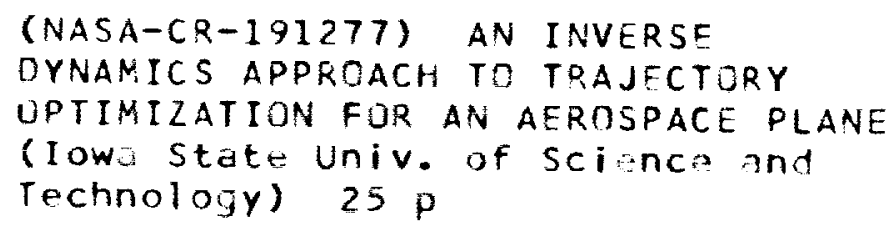

N93-13305

Unclas

$63 / 05 \quad 0131308$

* Assistant Professor, Department of Aerospace Engineering \& Engineering Mechanics, Member AIAA 


\section{Introduction}

The National Aerospace Plane (NASP) is a concept for a hypersonic vehicle that will take off horizontally and fly into low-earth orbit. The power plant of the NASP will consist of an airbreathing propulsion system such as a ramjets/scramjets, possibly augmented by a rocket. For the past few years the fuel-optimal ascent problem has been the center of research on trajectory optimization and guidance for the NASP. In Refs. 1-3, the energy state approximation and a singular perturbation method have been employed to obtain near-optimal solutions. They prove to be effective in developing guidance and propulsion control laws. Feedback linearization and variable structure control techniques are used in Refs. 4 and 5 to develop a guidance logic. Near-optimal guidance is obtained by tracking a near-optimal trajectory which is a reduced-order solution of the vehicle dynamics. It has been observed, however, that the validity of time scale decomposition needs careful examination for some portions of the flight envelope. Fuel-optimal trajectories are studied in Ref. 6 using a trajectory program, $\mathrm{POST}^{7}$, and some issues in guidance and flight control are addressed. Due to the complex nature of the optimization problem, it appears that enormous difficulty will be encountered if indirect methods based on the necessary conditions for optimality are to be used. When a realistic model of the NASP is used, direct methods based on nonlinear programming (NLP) are most promising for accurate off-line trajectory optimization. In addition, discontinuities due to propulsion system switchings, state-inequality constraints and possible singular control arcs are conveniently handled by such methods. But it has been found that even a conventional NLP approach in trajectory optimization in which controls are parametrized directly by certain functions encounters difficulty $^{8}$. This is because the NASP trajectory has high sensitivity to the controls. As a result, the optimization problem is poorly conditioned. A collocation algorithm (e.g., Ref. 9) appears to be a better choice for this problem ${ }^{5}$ because of its robustness. This paper considers an alternative, namely, an inverse dynamics approach. In this approach, the control is implicitly parameterized. This greatly improves the conditioning of the optimization problem, and allows us to obtain optimal trajectories and controls reliably via the nonlinear programming approach.

The remainder of this paper is organized as follows. The equations of motion and the model of an aerospace plane are described in Section 2. The trajectory optimization problem is formulated in Section 3. An inverse dynamics approach is proposed for general trajectory optimization problems. Inverse aerodynamic control, and rocket control laws, when rocket-assistance is used for ascent, are developed based on inverse dynamics concept. Using this approach, we obtain solutions of both minimum-fuel and minimum-peak 
dynamic pressure for the aerospace plane in Section 4. In Section 5, the inverse rocket control laws together with two guidance laws are shown to guarantee accurate orbital insertion in the presence of disturbances.

\section{Dynamics and Model of Aerospace Plane}

\subsection{Equations of Motion}

Assuming a spherical, nonrotating earth and gravitational field with $g=\mu / r^{2}$, we have the 2-D point mass equations of motion for the aerospace plane

$$
\begin{aligned}
\frac{d r}{d t} & =v \sin \gamma \\
\frac{d \theta}{d t} & =\frac{v \cos \gamma}{r} \\
\frac{d v}{d t} & =\frac{T \cos (\alpha-\varepsilon)-D}{m}-\frac{\mu \sin \gamma}{r^{2}} \\
\frac{d \gamma}{d t} & =\frac{T \sin (\alpha-\varepsilon)+L}{m v}+\left(\frac{v}{r}-\frac{\mu}{v r^{2}}\right) \cos \gamma \\
\frac{d m}{d t} & =-\frac{T}{g_{0} I_{s p}}
\end{aligned}
$$

In above equations, $r$ is the radius from the center of the earth to the vehicle; $\theta$ the polar angle; $v$ the velocity; $\gamma$ the flight path angle and $m$ the total mass. $T$ is the thrust and $I_{s p}$ is the specific impulse of the propulsion system. $\alpha$ denotes the angle of attack. $\varepsilon$ is the thrust vector angle and will be assumed to be constant or zero for airbreathing engines. $L$ and $D$ are aerodynamic lift and drag, respectively. The atmospheric density is assumed to be an exponential function of altitude,

$$
\rho=\rho_{0} e^{-\beta\left(r-r_{0}\right)}
$$

where $r_{0}=6378 \mathrm{~km}$ is the radius of the earth.

\subsection{Vehicle Model}

The model of the aerospace plane used in this paper is based on Ref. 10, known as the "Langley Accelerator". It is a winged-cone configuration with a reference area of $S_{r e f}=334.73 \mathrm{~m}^{2}\left(3603 \mathrm{ft}^{2}\right)$ and length of $60.96 \mathrm{~m}(200 \mathrm{ft})$. The gross takeoff mass is $136,077 \mathrm{~kg}(300,000 \mathrm{lbf})$. The thrust of the airbreathing propulsion system is given by

$$
T=C_{T} q
$$

where $q$ is the dynamic pressure, and $C_{T}$ is the thrust coefficient. $C_{T}$ and $I_{s p}$ are given in tabular form as functions of Mach number, dynamic pressure and fuel-equivalence ratio, 
designated by $\phi$ hereafter. Engine throttling is controlled by $\phi$. An equivalence ratio of unity corresponds to maximum fuel efficiency; values greater than unity give more thrust but use disproportionately more fuel.

The airframe lift and drag coefficients $C_{L}$ and $C_{D}$ are also given as functions of Mach number and the angle of attack in tabulated data. Throughout this paper $C_{L}$ for the basic vehicle is approximated by

$$
C_{L}=C_{L 1} \alpha+C_{L 3} \alpha^{3}
$$

where $C_{L 1}$ and $C_{L 3}$ are functions of Mach number, obtained by least-squares fittings to the tabulated data for given $\alpha$, and then interpolated by cubic splines over Mach number. The speed of sound is approximated by a cubic polynomial of altitude $h=r-r_{0}$ :

$$
V_{s}=a_{0}+a_{1} h+a_{2} h^{2}+a_{3} h^{3}
$$

The coefficients $a_{i}$ 's are least-squares fittings to the 1976 US standard atmosphere ${ }^{11}$.

\section{Trajectory Optimization}

\subsection{Problem Statement}

Assume the following horizontal takeoff conditions:

$$
\begin{aligned}
h(0) & =0 \\
\theta(0) & =0 \\
v(0) & =170 \mathrm{~m} / \mathrm{s}(\text { Mach } 0.5) \\
\gamma(0) & =0 \\
m(0) & =133,809 \mathrm{~kg}(295,000 \mathrm{bf})
\end{aligned}
$$

The terminal conditions are specified as follows

$$
\begin{aligned}
h\left(t_{f}\right) & =h_{f} \\
\gamma\left(t_{f}\right) & =0 \\
v\left(t_{f}\right) & =\sqrt{\frac{\mu}{r_{0}+h_{c}}}
\end{aligned}
$$

These equations correspond to the conditions for insertion into a circular orbit at altitude of $h_{f}$ if $h_{f}$ is above sensible atmosphere. Operational constraints on the trajectory must be imposed. Two of the most important constraints are

$$
q \leq q_{\max }
$$




$$
Q \leq Q_{\max }
$$

Equations (10) and (11) place constraints on dynamic pressure $q$ and heating rate $Q$ at a specified point of the vehicle. The trajectory optimization problem for minimum-fuel ascent can be stated as: Find the optimal control $\alpha^{*}(t)$ and $\phi^{*}(t), 0 \leq t \leq t_{f}^{*}$, such that Eqs. (8)-(11) are satisfied by the solution of the dynamic equations (1)-(5) and the final mass $m\left(t_{f}^{*}\right)$ is maximized.

It was pointed out in the introduction that to solve this optimal control problem with-

out further simplifications, direct methods using the nonlinear programming approach have obvious advantages. But earlier work has shown that even a conventional direct parametrization algorithm has difficulty in achieving convergence because of high sensitivity of hypersonic flight ${ }^{8,12}$. This motivates us to develop an alternative approach in the next section.

\subsection{An Inverse Dynamics Approach}

The concept of inverse dynamics has recently gained considerable popularity in aerospace controls, e.g., Refs. 13-16. The inverse dynamic problem (IDP) considered here can be formally stated as the following:

Given a dynamic system

$$
\dot{\mathbf{x}}=\mathbf{f}(\mathbf{x}(t), \mathbf{u}(t), t)
$$

where $\mathbf{x} \in R^{n}$ and $\mathbf{u} \in R^{m}$. Initial conditions and terminal constraints are

$$
\begin{gathered}
\mathbf{x}\left(t_{0}\right)=\mathbf{x}_{0} \\
\mathbf{S}\left(\mathbf{x}\left(t_{f}\right), t_{f}\right)=0
\end{gathered}
$$

Find a control $\mathbf{u}(t)$ such that the solution of (12) with initial condition (13) satisfies Eq. (14) and the algebraic constraint

$$
\mathbf{g}(\mathbf{x}(t), \mathbf{c}(t), t)=0, \quad t \in\left[t_{0}, t_{f}\right]
$$

where $\mathbf{g}: \mathbf{R}^{n} \times \mathbf{R}^{l} \times R \rightarrow \mathbf{R}^{l}$ is sufficiently differentiable. $\mathbf{c}(t) \in \mathbf{R}^{l}$ for $t_{0} \leq t \leq t_{f}$ is a given smooth function. $\mathbf{c}(t)$ usually represents desired outputs and (15) specifies the output relationship. By repeatedly differentiating each component of (15) until components of $\mathbf{u}$ appear explicitly, we have additionally

$$
\mathbf{G}(\mathbf{x}(t), \mathbf{u}(t), \mathbf{c}(t), \dot{\mathbf{c}}(t), \ldots, t)=0
$$

Equations (15) and (16) constitute constraints on the state variables and controls. Most work in this area has so far centered on finding the required control for a given output 
c $(t)$ (e.g., Ref. [16]). In this paper, we extend this idea to trajectory optimization. The thought is simple: If an optimal output $\mathbf{c}^{*}(t)$ is found, the corresponding control $\mathbf{u}^{*}(t)$ can be determined from Eq. (16). For a given trajectory optimization problem, one needs to define the relation Eq. (15) in order to implement the idea. While many possibilities may exist in doing so, the general guidelines are that the most influential variable(s) are chosen as the output(s), and the process of solving for controls from Eq. (16) is kept relatively simple.

\subsection{Inverse Aerodynamic Control}

To apply the concept of inverse dynamics more effectively to the current problem, we first change the independent variable from $t$ to $\theta$. The system equations (1)-(5) now become

$$
\begin{aligned}
\frac{d r}{d \theta} & =r \tan \gamma \\
\frac{d t}{d \theta} & =\frac{r}{v \cos \gamma} \\
\frac{d v}{d \theta} & =\left(\frac{T \cos \alpha-D}{m}-\frac{\mu \sin \gamma}{r^{2}}\right) \frac{r}{v \cos \gamma} \\
\frac{d \gamma}{d \theta} & =\left(\frac{T \sin \alpha+L}{m v}+\left(\frac{v}{r}-\frac{\mu}{v r^{2}}\right) \cos \gamma\right) \frac{r}{v \cos \gamma} \\
\frac{d m}{d \theta} & =-\frac{T}{g_{0} I_{s p}} \frac{r}{v \cos \gamma}
\end{aligned}
$$

where we have assumed that $\varepsilon=0$ for the airbreathing propulsion system, although this is not necessary for the following development. Analogous to (15), we define

$$
g=r(\theta)-c(\theta)=0
$$

In (22) $c(\theta)$ is a sufficiently smooth function that represents a specified altitude history. Differentiating (22) once with respect to $\theta$ gives

$$
\tan \gamma=\frac{c^{\prime}}{r}
$$

Differentiating (23) once again, we have

$$
L(\alpha)=m v\left[\left(c^{\prime \prime}-r \tan ^{2} \gamma\right) \frac{v \cos ^{3} \gamma}{r^{2}}-\left(\frac{v}{r}-\frac{\mu}{v r^{2}}\right) \cos \gamma-\frac{T \sin \alpha}{m v}\right]
$$

The prime in (23) and (24) stands for differentiation with respect to $\theta$. Equation (23) determines the required $\gamma$ for $r$ to follow $c(\theta)$. Equation (24) specifies the necessary lift 
control. For a specified thrust level $T$ and the current values of the state variables, Equation (24) constitutes an algebraic equation for $\alpha$. With $\alpha$ solved from (24), Equations (19) and (21) can be integrated for $v$ and $m$ at next instant. So the solution of the system as well as the value of the performance index (fuel consumption, for instance) is completely determined by the choice of the pair of command altitude $c$ and fuel-equivalence ratio $\phi$. If we choose to represent $c(\theta)$ and $\phi(\theta)$ by certain smooth parametrized functions, the optimization problem reduces to a parameter optimization problem in which the best $c$ and $\phi$ histories are iteratively sought through solving a sequence of inverse dynamic problems. Note that in the parametrization of $c$, one can always choose the boundary conditions

$$
\begin{gathered}
c\left(\theta_{f}\right)=r_{0}+h_{c} \\
c^{\prime}\left(\theta_{f}\right)=0
\end{gathered}
$$

Then the first two of the three terminal constraints in Eq. (9) are automatically satisfied according to (22) and (23), leaving only the constraint on $v_{f}$ to be met. With $C_{L}$ represented by Eq. (7), we can solve for $\alpha$ from Eq. (24) very effectively by Newton iterations with an accuracy of $10^{-6}$ frequently after only one iteration. Because of this and the fact that the $r$ and $\gamma$ equations need not be integrated, the computation of $\alpha$ does not pose an extra burden to the computer.

One of the main advantages of this inverse dynamics approach is that the trajectory is under more direct control of the parametrization process. Consequently, the sensitivity of the optimization problem is greatly reduced. In fact, with minimum efforts, one can easily construct various feasible trajectories that satisfy the terminal conditions (9) and state constraints (10) and (11) by choosing $c(\theta)$ and $\phi(\theta)$. This feature is not only essential to the success of the trajectory optimization, but may also be useful for quick design of hypersonic cruising trajectories (not necessarily optimal in any sense). Compared with the collocation method reported in Ref. 9, the current approach has the same merits such as improving conditioning of the problem and robustness of the algorithm. Because the state variables are not parametrized but obtained through integration, fewer optimization parameters may be used, which directly contributes to a faster convergence.

\subsection{Inverse Rocket Control}

When the orbital altitude is higher, the scramjets become ineffective during the final phase as the atmosphere gets thinner. A rocket will be needed. Calise et al show that along an optimal trajectory the scramjets may still stay on for the remaining flight after the rocket is turned on, although their contribution to thrust is insignificant ${ }^{1-2}$. From a practical point of view, the scramjets may be cut off at a selected point. If a throttleable rocket is 
used, the remaining rocket-assisted trajectory could consist of a combination of coasting, singular thrust and full-throttle arcs. We found that the fuel-consumption is not sensitive to the rocket throttle program at all. The reason is that after a long coast the rocket is turned on at approximately the orbital altitude. The optimal thrust angle $\varepsilon^{*}$ then is almost zero and the major role of the rocket is to increase the velocity to orbital speed. Since drag $D$ is very small at that altitude, the situation is similar to a rocket with fixed $\varepsilon$ in vacuumthe velocity increment is only dependent on the rocket fuel expenditure, independent of the throttle program. For this reason, we will not investigate the combination of singular and full-throttle arcs. Instead, we shall concentrate on variable-throttle control via the inverse dynamics approach.

Let $\alpha=0$ during the rocket thrusting (since aerodynamic lift is negligible at that altitude). Let the command altitude $c(\theta)$ and desired history of velocity $v(\theta)$ be specified for the rocket-assisted portion of the trajectory. In a similar way as Eq. (24) is derived (but now $\alpha=0$ and $\varepsilon \neq 0$ ), we have

$$
\begin{aligned}
T \sin \varepsilon & =\left(\frac{v}{r}-\frac{\mu}{v r^{2}}\right) m v \cos \gamma \\
& -\frac{m v^{2} \cos ^{3} \gamma}{r^{2}}\left(c^{\prime \prime}-r \tan ^{2} \gamma\right) \triangleq U \\
T \cos \varepsilon & =\frac{m v \cos \gamma}{r} v^{\prime}+\frac{\mu \sin \gamma}{r^{2}}+D \triangleq V
\end{aligned}
$$

Two resulting feedback control laws are

$$
\begin{aligned}
\eta & =\frac{1}{T_{\max }} \sqrt{U^{2}+V^{2}} \\
\tan \varepsilon & =\frac{U}{V}
\end{aligned}
$$

where $\eta$ represents the throttle of the rocket, and $T_{\max }$ the maximum available thrust. Instead of optimizing $c(\theta)$ and $v(\theta)$, we find it sufficient to let

$$
\begin{gathered}
c(\theta)=a+b \theta+d \theta^{2}+e \theta^{3} \\
v(\theta)=n+p \theta
\end{gathered}
$$

The duration of the rocket thrusting phase is an optimization parameter. The coefficients $a, b, d$ and $e$ in (31) are determined by continuities of $r$ and $\gamma$ at the instant $\theta_{r}$ when the rocket is turned on, and by Eqs. (25) and (26) for the first two terminal constraints in Eqs. (9). Coefficients $n$ and $p$ are defined by continuity of $v$ at $\theta_{r}$ and the final velocity constraint in (9). Since these calculations can be easily done onboard, the parametrizations 
(31) and (32) have a distinct advantage: The orbital insertion conditions (9) will remain satisfied even if the actual trajectory deviates from the nominal at $\theta_{r}$. This is because (31) and (32) always lead the trajectory from the states at $\theta_{r}$, whatever the values are, to the target point defined by Eqs. (9) when the coefficients are calculated with the actual values of the states at $\theta_{r}$. The corresponding inverse controls (29) and (30) are hence disturbance-accommodating, provided that they are not in violation of the control constraints $|\varepsilon| \leq \varepsilon_{\max }$ and $\eta \leq 1$. An application of this feature is demonstrated in Section 5 .

The complete ascent trajectory with rocket-assistance can be parametrized in the following sequence: Airbreathing-engine powered ascent portion as described in Section 3.3; a coasting arc following the cutoff of the scramjets with $\alpha=0$; rocket-assisted flight into orbit. The optimization parameters include all those used in Section 3.3 plus the durations of coast and rocket-assisted flight. Once the optimization process converges, such a trajectory can be qualified as a near-optimal solution.

\section{Optimal Solutions}

For numerical solutions, equivalence ratio $\phi$ and command $c$ are parametrized by cubic splines. Twenty nodes are used for the $c$-history and ten for $\phi . C_{D}, C_{T}$ and $I_{s p}$ are interpolated by a table look-up scheme. To gain some insight into the problem, let us first consider the state inequality constraint (10) only. A new variable $w_{q}$ is introduced such that

$$
w_{q}(0)=0, \quad \dot{w}_{q}= \begin{cases}-\left(q-q_{\max }\right), & q>q_{\max } \\ 0, & q \leq q_{\max }\end{cases}
$$

Constraint (10) is equivalent to the terminal constraint

$$
w_{q}\left(\theta_{f}\right) \geq 0
$$

A similar transformation can be done to constraint (11). The optimal control problem is thus converted into a nonlinear programming problem. A sequential quadratic programming (SQP) $\operatorname{code}^{17}$ is employed to find the optimal solution.

\subsection{Minimum-Fuel Solutions}

Extensive computations have been performed for ascent trajectories to a target altitude at $h_{f}=55 \mathrm{~km}$ using the airbreathing propulsion only. This altitude is chosen such that the thrust given by Eq. (6) is still adequate to control the vehicle to meet the terminal conditions (9). The constraint (10) is found to be absolutely necessary for a realistic fueloptimal trajectory, because along the unconstrained optimal trajectory the peak dynamic 
pressure can reach $4.25 \times 10^{6} \mathrm{~N} / \mathrm{m}^{2}\left(8.87 \times 10^{4} \mathrm{psf}\right)$. It is no surprise in view of the propulsion model (6). The unconstrained optimal trajectory will seek high dynamic pressure to accelerate the vehicle to the required final speed in a short time so as to reduce the drag loss. The final masses for the optimal trajectories corresponding to different constraint levels are listed in Table 1.

Table 1. Summary of Minimum-Fuel Solutions

$$
q \leq q_{\max }
$$

\begin{tabular}{|c|c|c|c|c|c|}
\hline$q_{\max }\left(\mathrm{N} / \mathrm{m}^{2}\right)$ & $\infty$ & 239,400 & 191,520 & 143,640 & 95,760 \\
\hline$m\left(t_{f}\right)(\mathrm{kg})$ & 69,859 & 68,781 & 68,525 & 68,101 & 66,351 \\
\hline
\end{tabular}

Figure 1 depicts the ascent history $h(t)$ for $q_{\max }=95,760 \mathrm{~N} / \mathrm{m}^{2}(2000 \mathrm{psf})$. Shown in Fig. 2 are $\alpha(t)$ and $\gamma(t)$ for the same trajectory The optimal trajectory can be divided into three obvious segments: initial climb out, midcourse cruise during which the constraint $q \leq q_{\max }$ is active, and final zoom to the target altitude. The midcourse portion generally exhibits characteristics similar to those reported in the literature ${ }^{1-6}$. It features small angle of attack, small flight path angle, and active state constraint $q=q_{\max }$. References 3 and 6 also use the same vehicle model as in this paper but the aerodynamic control is through elevon deflection. Yet the angle of attack history in Ref. 6 is very similar to ours in Fig. 2: the initial climb requires large $\alpha$ to generate the needed lift, then $\alpha$ quickly becomes small. Also in Fig. 2, we see that large initial $\gamma$ helps achieve a quick climb and keep the trajectory within the region where $q \leq q_{\max }$. What has not been seen reported is an interesting feature of the optimal equivalence ratio $\phi$. Figure 3 shows equivalence ratio histories along three optimal trajectories. Observe that for the two trajectories with the dynamic pressure constraint, the optimal equivalence ratio is unity for a dominant portion which corresponds to the best fuel efficiency. For the unconstrained $\left(q_{\max }=\infty\right)$ and less constrained $\left(q_{\max }=191,520 \mathrm{~N} / \mathrm{m}^{2}\right)$ case, the final maneuver for satisfaction of the terminal constraints uses larger $\phi$. When the constraint on $q$ gets tighter, $\phi$ stays almost unity after the initial adjustment.

In the constrained case, the terminal constraint (34) prevents violation of the dynamic pressure constraint $q \leq q_{m a x}$. This work and previous work ${ }^{18}$ show that more nodes in the parametrization of $c(\theta)$ result in better tracking of the constraint boundary $q=q_{\max }$. Alternatively, since the optimal ascent trajectory is known to climb on the boundaries of the constraints (10) and (11) subsequently ${ }^{1,2}$, the nonlinear controllers developed in Refs. 1-5 may be used to track these boundaries accurately. 


\subsection{Minimax Dynamic Pressure Solution}

It is seen from Table 1 that as the state constraint $q \leq q_{\max }$ gets tighter with smaller $q_{\max }$, the fuel consumption increases noticeably. Therefore, it is of practical interest to know what is the minimum constraint level $q_{\max }$ under which the aerospace plane can still achieve the specified final speed. This information certainly is valuable in evaluating the performance of the vehicle. It is also important to its structural design since this minimum $q_{m a x}$ reflects a minimum structural strength requirement. The problem of finding minimum $q_{\max }$ falls into the category of so-called minimax optimal control problems. The statement of this problem is

$$
\min _{\alpha(t), \phi(t)} J=\min _{\alpha(t), \phi(t)}\left\{\max _{t_{0} \leq t \leq t_{j}} q(t)\right\}
$$

subject to system equations (1)-(5), boundary conditions (8)-(9), and additional constraint

$$
m\left(t_{f}\right) \geq m_{\min }
$$

where $m_{\min }$ is the mass of the aerospace plane excluding fuel. For our model, $m_{\min }=$ $63,503 \mathrm{~kg}(140,000 \mathrm{lbf})$. Necessary conditions for optimality exist for such a type of minimax problems ${ }^{19}$. Because the necessary conditions generally lead to a multi-point boundary value problem, the application of the necessary conditions is not deemed realistic for this complex system. However, within the framework of our current approach, the problem can be readily transformed into the following one:

$$
\min q_{\max }
$$

subject to system equations (16)-(20), boundary conditions (8)-(9), constraint (36), and

$$
q \leq q_{\max }
$$

Constraint (38) is handled by the transformation shown in (33)-(34), $q_{\max }$ is treated as one more component of the parameter vector to be optimized. The inverse dynamics approach described above again proves effective in solving this problem. For our model and the given takeoff and final conditions (9) at $h_{f}=55 \mathrm{~km}$, the minimum $q_{\max }$ turned out to be $71,772 \mathrm{~N} / \mathrm{m}^{2}(1,499 \mathrm{psf})$, and the final mass is equal to $m_{\min }$. The variation of the minimax $q$-history is plotted in Fig. 4. The state and control histories have the same characteristics as mentioned in Section 4.1. It is seen from Fig. 4 that in order to build up the required final velocity with this lower $q_{\max }$, the flight time is increased by $40 \%$ as compared to the case where $q_{\max }=95,769 \mathrm{~N} / \mathrm{m}^{2}$, and about $80 \%$ of the trajectory stays on the boundary $q=q_{\max }$. All these can be directly attributed to the fact that the thrust 
of the airbreathing engines is proportional to dynamic pressure [Eq. (6)]. On the other hand, for our aerospace plane model, satisfaction of $q \leq q_{\max }$ for $q_{\max }$ lower than 71,772 $\mathrm{N} / \mathrm{m}^{2}$ will require more fuel than it carries to achieve the specified final velocity.

\section{Guaranteed Orbital Insertion with Rocket-Assistance}

In Section 3.4 inverse control laws were developed for the rocket, and the planning of the near-optimal ascent trajectory was described. Let the heating rate constraint (11) be modeled by

$$
Q=\left(4.919 \times 10^{-8}\right) \rho^{0.5} v^{3.0}
$$

In (39) density $\rho$ is in $\mathrm{kg} / \mathrm{m}^{3}$; velocity $v$ in $\mathrm{m} / \mathrm{sec}$ and $Q$ in Watt $/ \mathrm{cm}^{2}$. Equation (39) corresponds to equilibrium conditions on the surface of a wing leading edge $10 \mathrm{~cm}$ in radius $^{1-3}$. For $h_{f}=92.6 \mathrm{~km}(50 \mathrm{~nm})$, the near-optimal trajectory is plotted in Fig. 5 . The points of interest are marked. In particular, the scramjets are found to be turned off at $70 \mathrm{~km}$. Following a coast of 452 seconds, a throttleable rocket with $T_{\max }=266,893 \mathrm{~N}$ $(60,000 \mathrm{lbf})$ and $I_{s p}=440$ seconds starts firing at $92.3 \mathrm{~km}$ with an intermediate throttle of about 0.21 . Orbital insertion is achieved 88.5 seconds after the rocket ignition. The final mass is $64,931 \mathrm{~kg}$, which is comparable to $m_{f}=65,370$ previously obtained for $h_{f}=55$ $\mathrm{km}$ without rocket-assistance. It should be noted that the long coasting arc is found to be very beneficial to saving fuel (more than $3,000 \mathrm{~kg}$ ) as compared to the case where no coasting is allowed.

Once a nominal (optimal) trajectory for the aerospace plane is established, guidance laws are used to restore the flight path should deviations occur in the actual flight. Two guidance laws are proposed as follows

$$
\begin{gathered}
\alpha=\tilde{\alpha}-k_{h}\left(h-h^{*}\right)-k_{\gamma}\left(\gamma-\gamma^{*}\right), \quad k_{h}>0, \quad k_{\gamma}>0 \\
\phi=\phi^{*}-k_{v}\left(v-v^{*}\right), \quad k_{v}>0
\end{gathered}
$$

where the asterisk denotes the nominal values, values without asterisk are the actual values, measured or estimated. $\tilde{\alpha}$ in Eq. (40) is obtained by solving the following equation on-line

$$
L(\tilde{\alpha})=m v\left[\frac{\left(c^{*} c^{* \prime \prime}-c^{* / 2}\right)}{\left(c^{* 2}+c^{* / 2}\right)} \frac{v \cos \gamma}{r}-\left(\frac{v}{r}-\frac{\mu}{v r^{2}}\right) \cos \gamma-\frac{T \sin \tilde{\alpha}}{m v}\right]
$$

We note that Eq. (42) is the same as Eq. (24) if all the quantities are equal to the nominal values, that is, when there are no perturbations, $\alpha=\tilde{\alpha}=\alpha^{*}$.

Equation (41) provides a negative feedback to the throttle which tends to reduce the error in velocity. The rationale for Eq. (40) is explained in the following. Let $\alpha=\tilde{\alpha}+\Delta \alpha$. 
Linearizing the right hand side of the flight path angle dynamics [Eq. (20)] with respect to $\alpha$ produces

$$
\gamma^{\prime}=\left(\frac{T \sin \tilde{\alpha}+L(\tilde{\alpha})}{m v}+\left(\frac{v}{r}-\frac{\mu}{v r^{2}}\right) \cos \gamma\right) \frac{r}{v \cos \gamma}+\left(\frac{T \cos \tilde{\alpha}+q S_{r e f} \partial C_{L} / \partial \alpha}{m v}\right) \frac{r \Delta \alpha}{v \cos \gamma}
$$

With $L(\tilde{\alpha})$ given by Eq. (42), the first term in Eq. (43) reduces to the term $\left(c^{*} c^{* \prime \prime}-\right.$ $\left.c^{* \prime 2}\right) /\left(c^{* 2}+c^{* 2}\right)$ which by Eqs. (22) and (23) can be shown to be equal to $\gamma^{* \prime}$. So, Eq. (43) becomes

$$
\Delta \gamma^{\prime}=\left(\frac{T \cos \tilde{\alpha}+q S_{r e f} \partial C_{L} / \partial \alpha}{m v}\right) \frac{r}{v \cos \gamma} \Delta \alpha \triangleq w \Delta \alpha
$$

where $w>0$ is time-varying. By Eq. (40) $\Delta \alpha=-k_{h} \Delta h-k_{\gamma} \Delta \gamma$.

Thus,

$$
\Delta \gamma^{\prime}=-w k_{h} \Delta r-w k_{\gamma} \Delta \gamma
$$

where $\Delta r=\Delta h$ has been used. When some system parameters have variations known to the onboard computer, Eq. (45) indicates that $\tilde{\alpha}$ computed from Eq. (42) with the true values of the system parameters will result in $\Delta \gamma^{\prime}=0$ thus $\gamma=\gamma^{*}$ and $r=r^{*}$ if there are no initial errors. Next, assuming $\tan \gamma^{*} \approx \gamma^{*}$, we consider the linearized altitude dynamics

$$
\Delta r^{\prime}=\gamma^{*} \Delta r+r^{*} \Delta \gamma
$$

It is known that $\gamma^{* \prime} \approx 0$ and $\gamma^{*}$ is small for most part of the trajectory ${ }^{1-5,8}$. Using this approximation and differentiating Eq. (46) once with respect to $\theta$, we have

$$
\Delta r^{\prime \prime}-2 \gamma^{*} \Delta r^{\prime}-r^{*} \Delta \gamma^{\prime}=0
$$

If we let $k_{h}=\left(2 \gamma^{*}+k_{1}\right) / w$ and $k_{\gamma}=\left(k_{1} \gamma^{*}+k_{2}\right) / w r^{*}$, where $k_{1}>0$ and $k_{2}>0$ are two constants, Eqs. (45) and (47) lead to

$$
\Delta r^{\prime \prime}+k_{1} \Delta r^{\prime}+k_{2} \Delta r=0
$$

Then $\Delta r \rightarrow 0$ asymptotically.

The above analysis establishes asymptotic tracking of altitude by using control law (40) if $k_{h}$ and $k_{\gamma}$ are properly chosen to be functions of state variables. The key is $\tilde{\alpha}$ determined from Eq. (42) which leads to Eq. (44) without using linearization of the flight path angle dynamics with respect to the state and throttle.

To demonstrate the guaranteed accurate orbital insertion with the aid of the guidance laws (40) and (41), and the inverse control of the rocket in the presence of disturbances, we introduce two types of atmospheric density fluctuations. One is a vertical variation 


$$
\rho=\left(1+0.2 \sin \frac{2 \pi h}{50}\right) \rho^{*}
$$

In (49) $\rho^{*}$ is the nominal density. $h$ is altitude in $\mathrm{km}$. The maximum fluctuation of $20 \%$ as given by (49) is a modest variation of the density compared with the flight data of the Space Shuttle ${ }^{20}$. The other is a horizontal fluctuation

$$
\rho=\left(1+0.2 \sin \frac{2 \pi d}{1000}\right) \rho^{*}
$$

where in (49) $d=r_{0} \theta$ is the down range distance $(\mathrm{km})$. The nominal trajectory is the nearoptimal rocket-assisted trajectory in Fig. 5. The airbreathing propulsion portion of the trajectory is controlled by the guidance laws (40) and (41). These measures greatly reduce the deviations of the perturbed trajectories, which prevents the trajectories from crashing or entering regions where no feasible maneuvers of the rocket can restore the flight path (In fact, without compensation of (40) and (41), the trajectories would have crashed during the climb-out). Then after the coast the inverse rocket control laws (29) and (30) take over. Despite the deviations at this point from the nominal due to the atmospheric disturbances, the control laws still steer the aerospace plane to an accurate orbital insertion, assuming that the coefficients in Eqs. (29) and (30) are calculated onboard. Figure 6 depicts the nominal and the two perturbed trajectories. The final masses of the perturbed trajectories are $64,635 \mathrm{~kg}$ and $64,202 \mathrm{~kg}$, respectively. Clearly, the longitudinal fluctuation in $\rho$ affects the trajectory more adversely than the vertical fluctuation. This is no surprise if one compares the down range distance the aerospace plane travels (about $14,300 \mathrm{~km}$ ) with the altitude gained $(92.6 \mathrm{~km})$. The nominal and perturbed rocket throttle settings $\eta$ and thrust angles $\varepsilon$ are shown in Figs. 7 and 8 . The effects of the controls responding to disturbances are clearly seen.

\section{Concluding Remarks}

The trajectory optimization problem for an aerospace plane has been formulated as an inverse dynamic optimization problem. The method has proven quite effective in solving this otherwise very difficult problem. The approach of using inverse dynamics concept for trajectory optimization may be considered a valuable addition to the existing tool box for trajectory optimization, and used in other problems.

The performance indices considered in this paper are minimum fuel consumption and minimum peak dynamic pressure. A transform technique is used to solve the nonclassical minimax problem effectively within the framework of parameter optimization. Both problems are solved reliably using the inverse dynamics approach. The results lead to a better understanding of the characteristics of hypersonic flight. Rocket-assisted trajectories are 
also investigated. Rocket control laws based on inverse dynamics are developed that, together with two ascent guidance schemes, guarantee accurate orbital insertion even in the presence of disturbances and perturbations.

\section{Acknowledgment}

This research was supported by NASA Langley Research Center under Contract No. NAG-1-1255 for which Dr. Daniel D. Moerder is the the technical monitor.

\section{References}

${ }^{1}$ Calise, A. J., Corban, J. E., and Flandro, G. A., "Trajectory Optimization and Guidance Law Development for National Aerospace Plane Applications", Final Report, NASA CR Number NAG-1-784, Dec., 1988.

2 Corban, J. E., Calise, A. J, and Flandro, G. A., "Rapid Near-Optimal Aerospace Plane Trajectory Generation and Guidance", Journal of Guidance, Control, and Dynamics, Vol. 14, No. 6, November-December, 1991, pp. 1181-1190.

3 Moerder, D. D., Pamadi, B., and Dutton, K., "Constrained Energy State Suboptimal Control Analysis of a Winged-Cone Aero-Space Plane Concept", AIAA-91-5053, Third AIA A International Aerospace Planes Conference, Orlando, FL, 3-5, December, 1991.

4 Van Buren, M. A., and Mease, K. D., "Aerospace Plane Guidance Using Geometric Control Theory", Proceedings of the 1990 American Control Conference, vol. 2, San Diego, CA, May, 1990.

5 Van Buren, M. A., and Mease, K. D., "Aerospace Plane Guidance Using Time-Scale Decomposition and Feedback Linearization", AIAA Journal of Guidance, Control, and Dynamics, Vol. 15, No. 5, Sept.-Oct., 1992, pp. 1166-1174.

${ }^{6}$ Powell, R. W., Shaughnessy, J. D., Cruz, C. I., and Naftel, J. C., "Ascent Performance of an Air-Breathing Horizontal-Takeoff Launch Vehicle", Journal of Guidance, Control, and Dynamics, Vol. 14, No. 4, July-August, 1991, pp. 834-839.

7 Brauer, G. L., Cornick, D. E., and Stevenson, R., "Capabilities and Applications of the Program to Optimize Simulated Trajectories (POST)', NASA CR-2770, Feb., 1977

${ }^{8} \mathrm{Lu}$, P., "Trajectory Optimization and Guidance for a Hypersonic Vehicle", AIAA-915068, Third AIAA International Aerospace Planes Conference, Orlando, FL, 3-5, December, 1991. 
${ }^{9}$ Hargraves C. R., and Paris S. W., " Direct Trajectory Optimization Using Nonlinear Programming and Collocation", Journal of Guidance, Control, and Dynamics, Vol. 10, No. 4, pp. 338-342, 1987.

${ }^{10}$ Shaughnessy, J. D., Pinckey, S. Z., McMinn J. D., Cruz, C. I., and Kelley M-L., "Hypersonic Vehicle Simulation Model: Winged-Cone Configuration", NASA TM 102610, November 1990 .

11 U.S. Standard Atmosphere, 1976, U.S. Government Printing Office, 1976.

12 Gilbert, E. G., Howe, R. M., Lu, P. and Vinh, N. X., "Optimal Aeroassisted Intercept Trajectories At Hyperbolic Speeds", Journal of Guidance, Control, and Dynamics, Vol. 14, No. 1, Jan.-Feb., 1991, pp. 123-131.

${ }^{13}$ Sentoh E., and Bryson, A.E., "A Comparison of Inverse Control with Optimal Control", Proceedings of AIAA Guidance, Navigation, and Control Conference, Portland, OR, August, 1990

${ }^{14}$ Lane, S. H., and Stengel, R. F., "Flight Control Design Using Nonlinear Inverse Dynamics", Automatica, Vol. 24, No. 4, July, 1988, pp. 471-483.

${ }^{15}$ Menon, P. K. A., Badgett, M. E., and Walker, R. A., "Nonlinear Flight Test Trajectory Controllers for Aircraft", Proceedings of AIAA Guidance, Navigation, and Control Conference, Snow Mass, CO, August, 1985.

${ }^{16}$ Vukobtatovic, M., and Stojic R., Modern Aircraft Flight Control, Springer-Verlag, New York, 1988.

${ }^{17}$ Pouliot, M. R., "CONOPT2: A Rapidly Convergent Constrained Trajectory Optimization Program for TRAJEX", Report N0. GDC-SP-82-008, General Dynamics, Convair Division, San Diego, CA, 1982.

${ }^{18} \mathrm{Lu}, \mathrm{P}$. , "Trajectory Optimization and Guidance for an Advanced Launch Vehicle", AIAA-92-0732, AIAA 30th Aerospace Sciences Meetings \& Exhibit, Jan. 6-9, Reno, NV.

${ }^{19} \mathrm{Lu}$, P. and Vinh, N. X., "Optimal Control Problems with Maximum Functional", Journal of Guidance, Control, and Dynamics, Vol. 14, No. 6, November-December, 1991, pp. $1215-1223$.

${ }^{20}$ Findlay, J.T., Kelly, G.M., McConnell, J.G., and Compton, H.R., "Shuttle 'Challenger' Aerodynamic Performance From Flight Data Comparison with Predicted Values and 'Columbia' Experience", AIAA paper 84-0485, Jan., 1984. 


\section{List of Captions}

Fig. 1 Optimal ascent history $\left(q \leq 95,760 \mathrm{~N} / \mathrm{m}^{2}\right)$

Fig. 2 Variations of optimal fuel-equivalence ratios

Fig. 3 Histories of flight path angle $\gamma$ and angle of attack $\alpha\left(q \leq 95,760 \mathrm{~N} / \mathrm{m}^{2}\right)$

Fig. 4 Variation of dynamic pressure along the min- $q_{\max }$ solution

Fig. 5 Ascent altitude history of a rocket-assisted trajectory $\left(q \leq 95,760 \mathrm{~N} / \mathrm{m}^{2}, Q \leq 800 \mathrm{~W} / \mathrm{cm}^{2}\right)$

Fig. 6 Comparison of nominal and perturbed trajectories in the presence of vertical atmospheric density fluctuation (perturbed I) and horizontal fluctuation (perturbed II).

Fig.7 Comparison of nominal and perturbed rocket throttle settings in the presence of vertical atmospheric density fluctuation (perturbed I) and horizontal fluctuation (perturbed II).

Fig.8 Comparison of nominal and perturbed rocket thrust angles in the presence of vertical atmospheric density fluctuation (perturbed I) and horizontal fluctuation (perturbed II). 


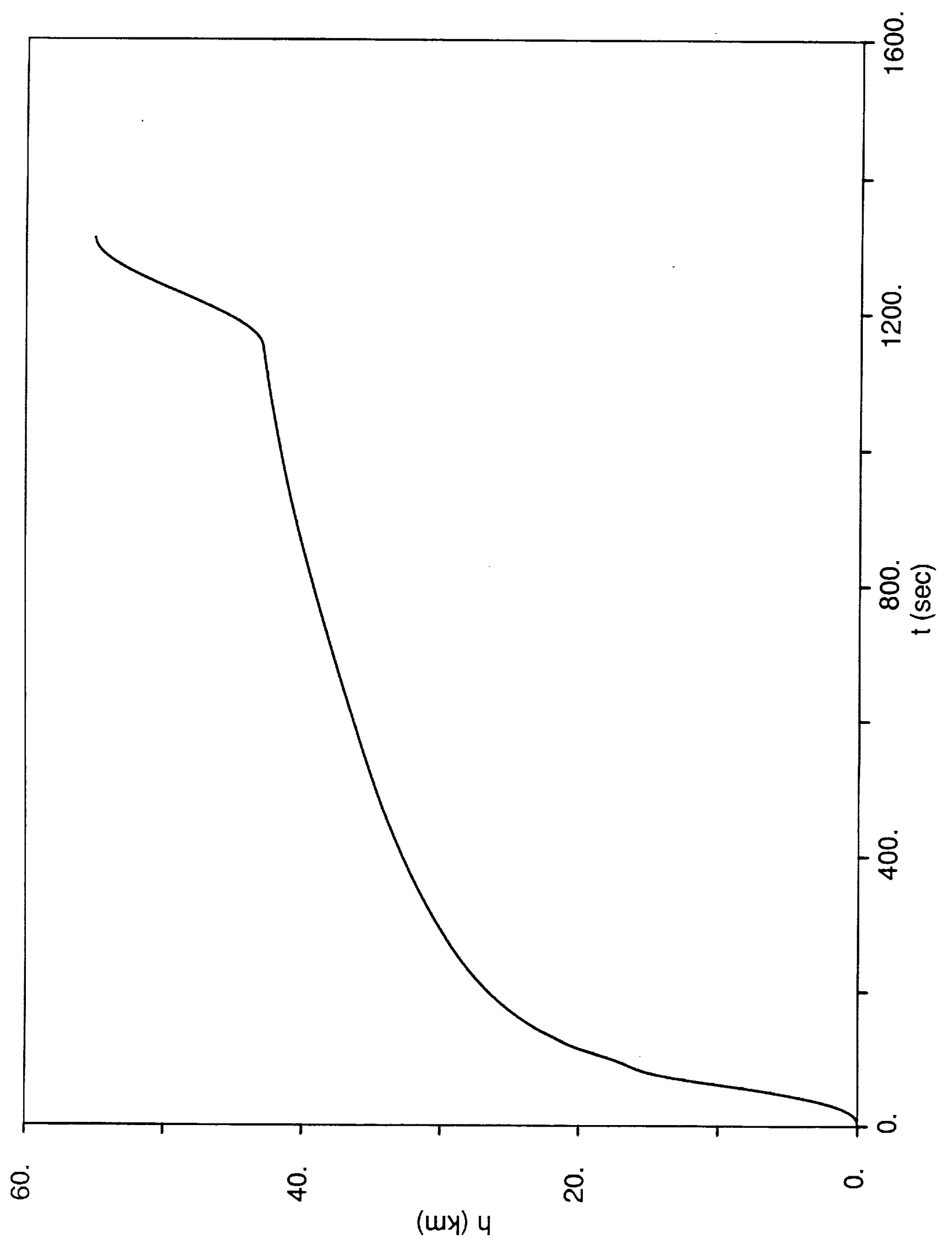




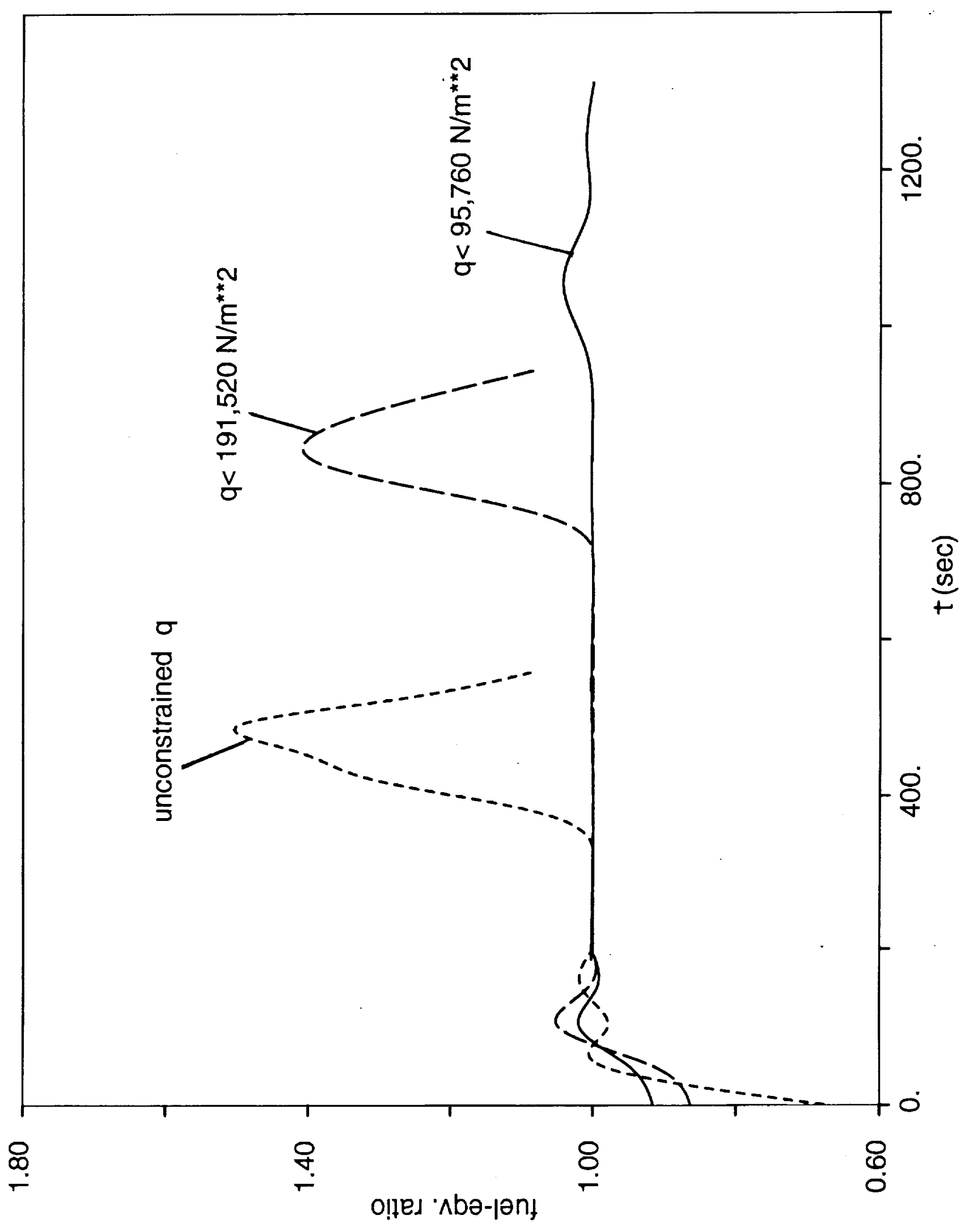


$m$
4

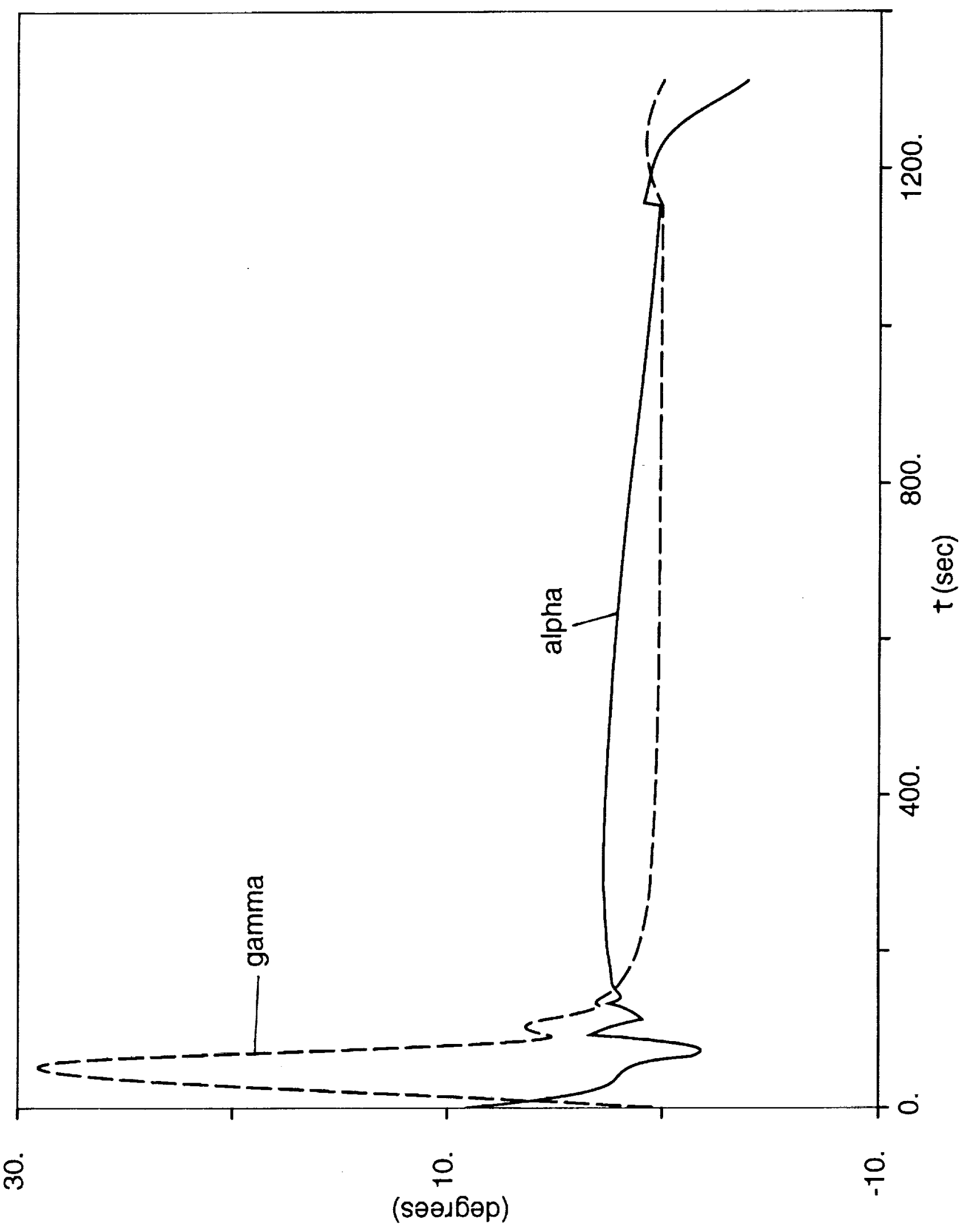




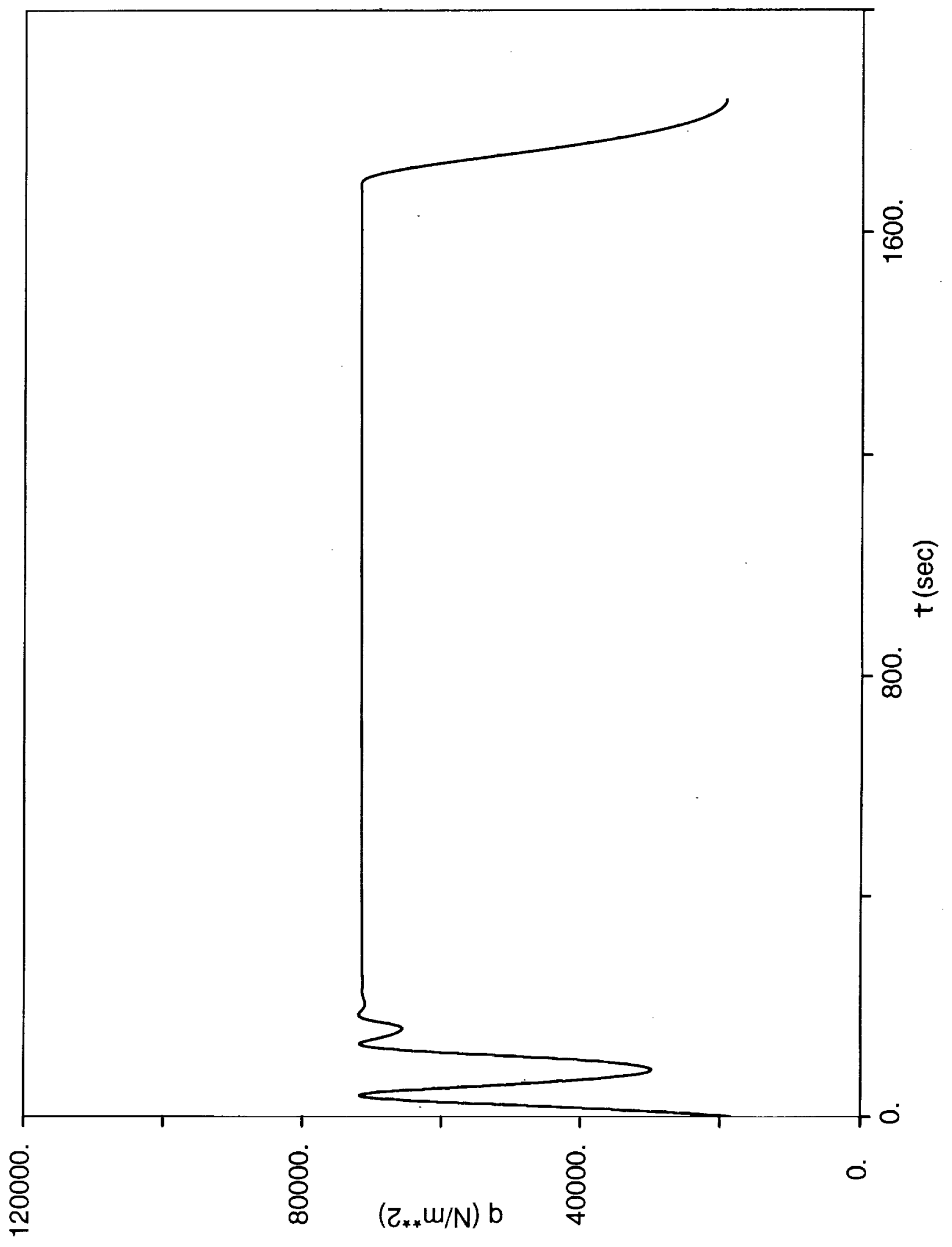




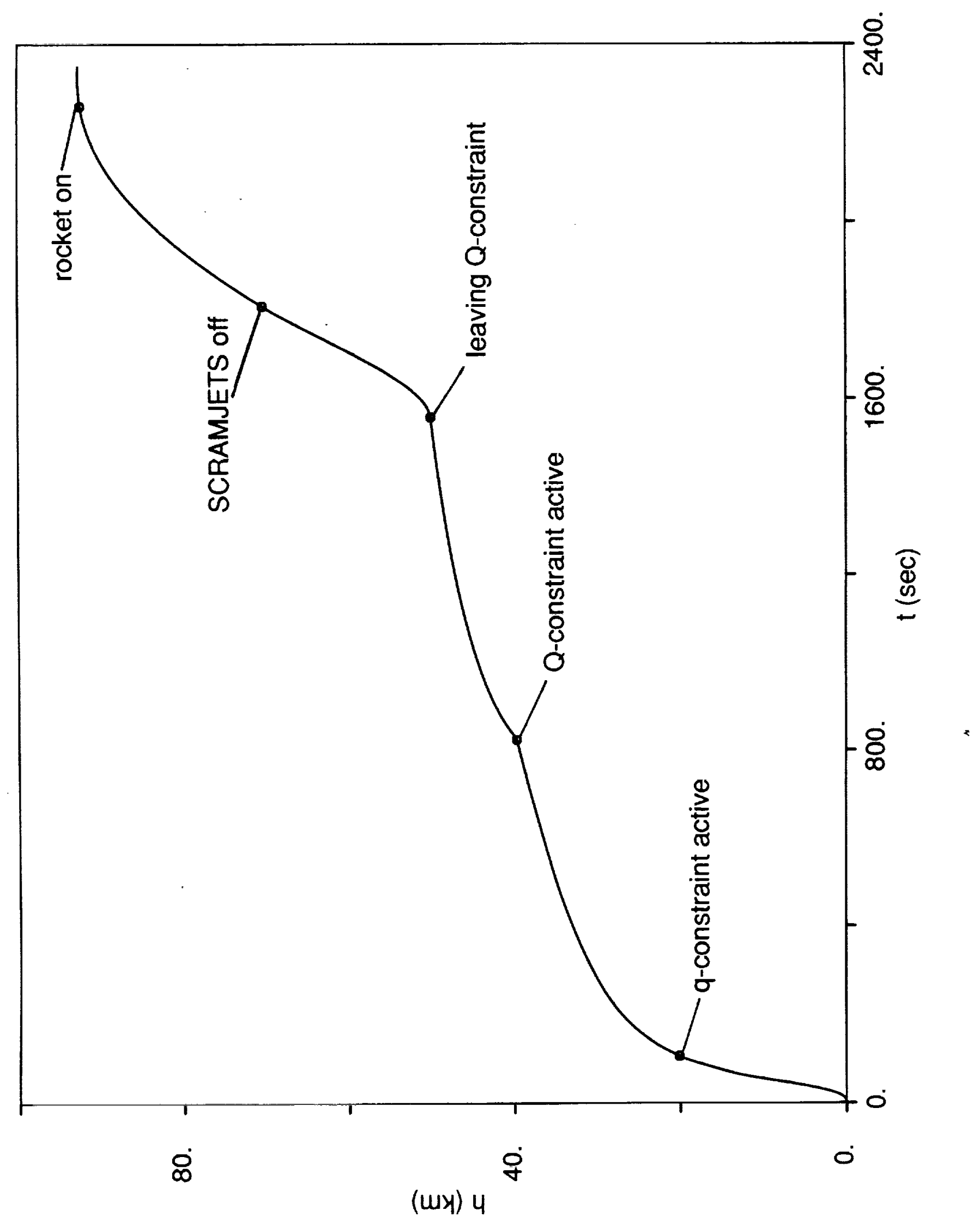




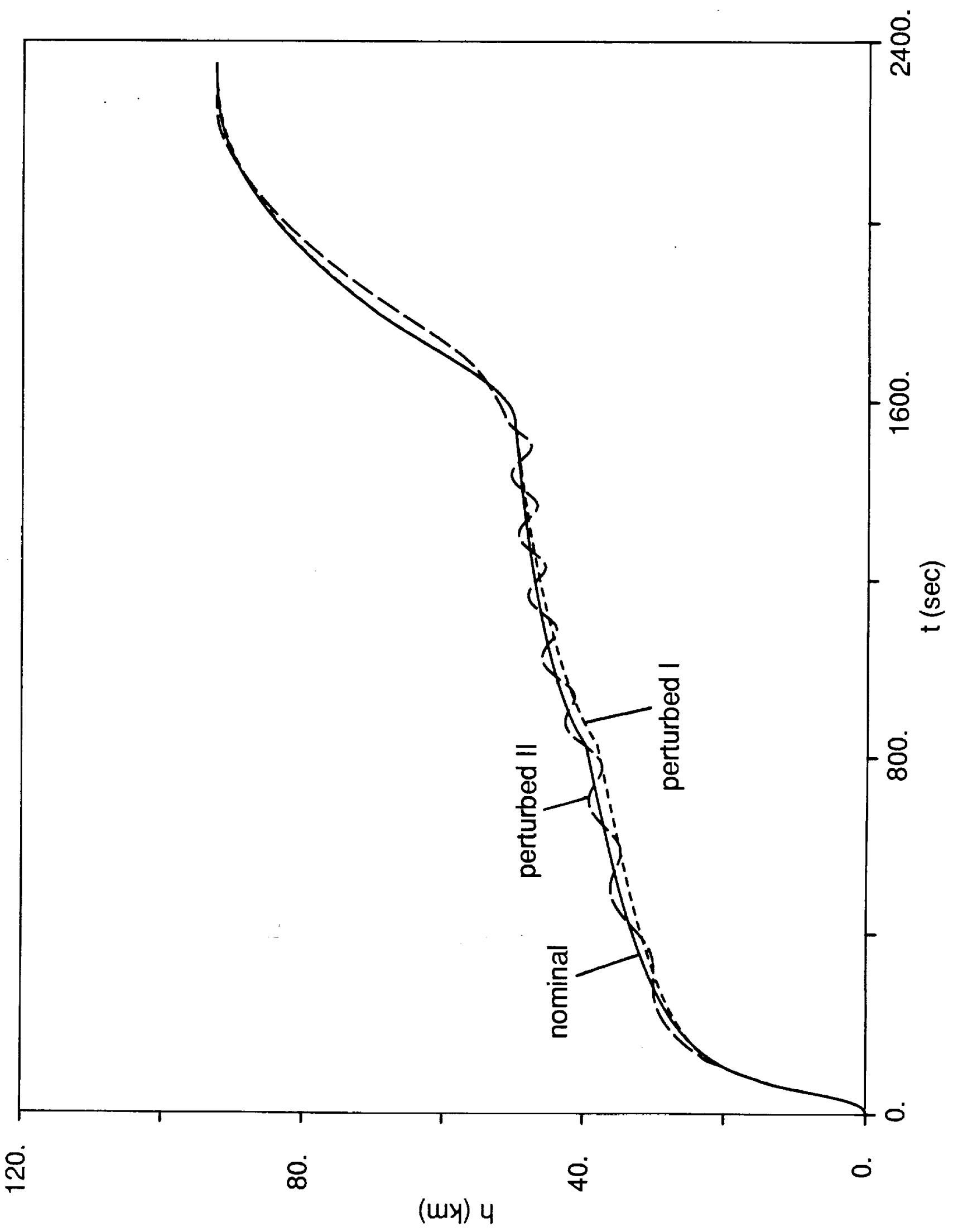




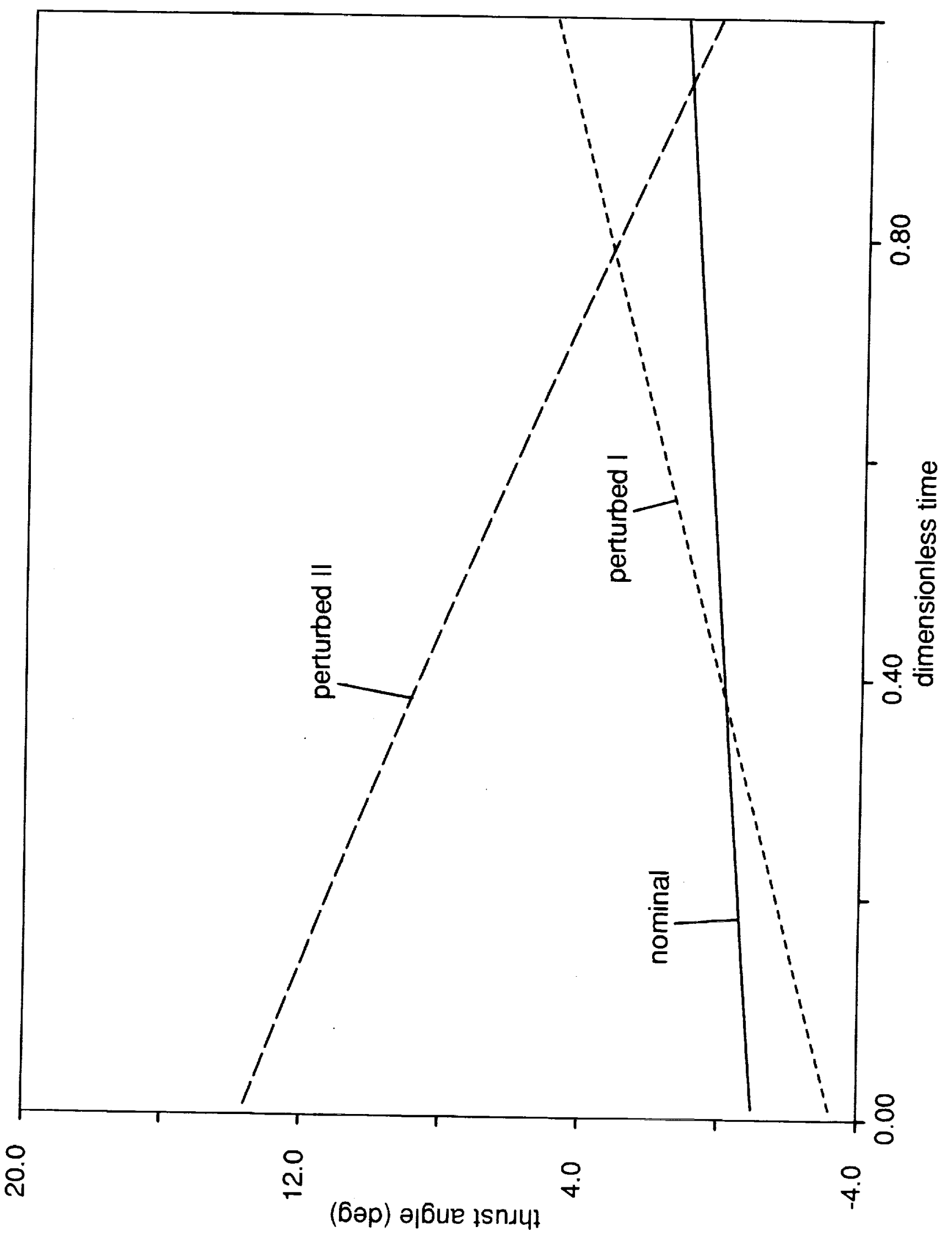




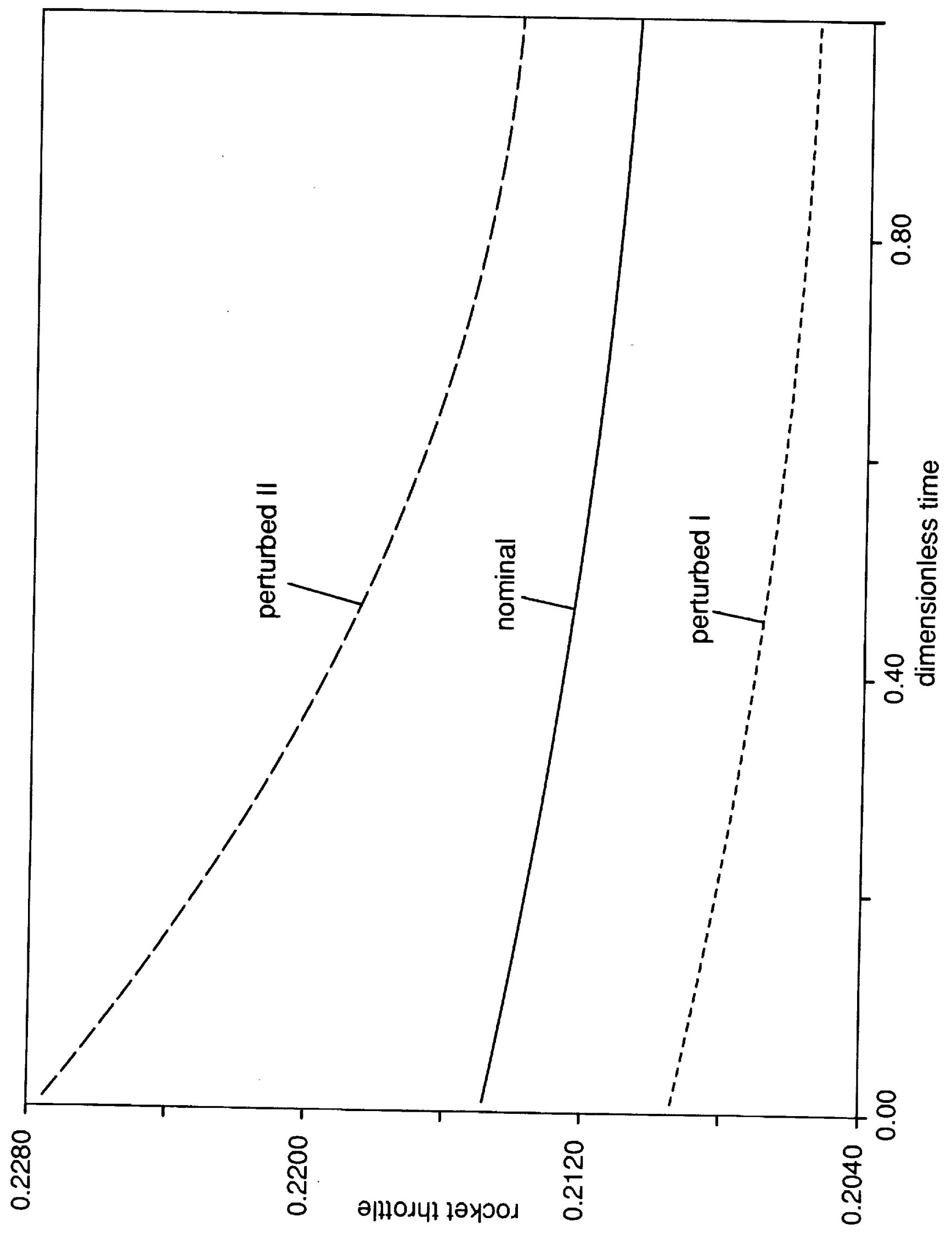


